\title{
Chronic Vaginal Malodor in the Absence of Infection: A Possible Manifestation of Olfactory Reference Syndrome
}

\author{
Machefsky Aliza ${ }^{1,2 *}$, Miller Collin², Smith Katharine T ${ }^{1}$ and Nyirjesy Paul ${ }^{1}$ \\ ${ }^{1}$ Department of Obstetrics and Gynecology, Drexel University, USA \\ ${ }^{2}$ Department of Obstetrics, Gynecology and Women's Health, St. Louis University, USA
}

Submission: December 06, 2018; Published: December 17, 2018

*Corresponding author: Aliza Machefsky, Department of Obstetrics and Gynecology, Drexel University, USA and Department of Obstetrics, Gynecology and Women's Health, St. Louis University, USA .

Abstract

Background: While bacterial vaginosis is a common cause of vaginal malodor, other women with vaginal malodor may have no objective findings. These women present a challenge since there is no satisfactory diagnosis or treatment. Olfactory reference syndrome is a psychiatric disorder characterized by a preoccupation with the belief that there is a offensive body odor, often from the genital region.

Objective: We studied women with chronic vaginal malodor and no infection, as they may have olfactory reference syndrome.

Study design: We performed a case-control study of 50 women with vaginal malodor and 51 women with recurrent bacterial vaginosis. All patients underwent a standardized evaluation at The Drexel Vaginitis Center--a tertiary care program established to evaluate women with chronic vulvovaginal symptoms. Historical and clinical variables were collected. Differences in demographic characteristics, medical/ psychological comorbidities, and symptoms were assessed.

Results: The median age was 36.5 years in cases vs. 33.0 in controls. 20 (40.0\%) vs. $15(29.4 \%)$ were white. $8(16 \%)$ vs. 5 (9.8\%) were menopausal. Both groups had similar rates of diagnosed psychiatric conditions ( $8 \%$ vs $7.8 \%$ ). The median duration of symptoms was 2 years vs. 1 year. All cases and 38 (74.5\%) of controls complained of malodor, described most commonly as fishy. Vaginal malodor patients were more likely to associate their odor with sweat $10(20 \%)$ vs. $0(\mathrm{P}=0.001)$ and complain that others noted the odor $10(20 \%)$ vs. $0(\mathrm{P}=0.001)$. $46(92 \%)$ of the malodor and $40(78.4 \%)$ of the bacterial vaginosis group had been previously diagnosed with bacterial vaginosis. After the initial evaluation showed no infection, 31 (63.3\%) vaginal malodor patients attempted some sort of intervention, with 18 (78.3\%) reporting no change.

Conclusions: Women with vaginal malodor are demographically similar to women with recurrent bacterial vaginosis. Standard interventions to address abnormal odor are frequently unhelpful. These women may have a manifestation of ORS.

Keywords: Bacterial vaginosis; Olfactory reference syndrome; Vaginal malodor

Abbrevations: BV: Bacterial Vaginosis; VM: Vaginal Malodor; ORS: Olfactory Reference Syndrome; IQR: Interquartile Range

\section{Introduction}

Vaginal malodor (VM) is a frequent complaint in the OB/ GYN office. While many patients with this complaint may have common infectious etiologies, especially bacterial vaginosis (BV), approximately one third of women presenting with the complaint of malodor do not have an identifiable cause [1]. While there are currently many studies describing vaginal odor in the setting of vaginal infections, there are little data exploring VM in the setting of noninfectious causes [2,3,4]. Included among these women are those who present with a complaint of VM and have no objective or clinician-documented odor. These women present a challenge since there is no satisfactory diagnosis or treatment option for these women [1].

Olfactory reference syndrome (ORS) is a psychiatric condition characterized by persistent preoccupation with the belief that one emits a foul or offensive body odor, often from the skin, mouth, rectum or genitals, that is not perceived by others. It is accompanied by shame, embarrassment, significant distress, avoidance behavior and social isolation [5]. In addition, it is often accompanied by delusions of reference (belief that others recognize and react to the aversive smell) and repetitive 
behaviors to mask the smell such as showering excessively and changing clothes multiple times throughout the day, or repetitively smelling themselves $[5,6,7,8]$. While ORS has been discussed in the psychiatric literature since the 1890s, and descriptions of the disorder have been found around the world, ORS remains an underdiagnosed and poorly understood disorder [5]. What little data exist on the condition suggests that affected individuals often present to non-psychiatric clinicians for help $[6,7]$. It is possible that many women who present to their OB/ GYN with the complaint of VM without an identifiable cause may have ORS.

At the Drexel Vaginitis Center, a tertiary care center that specializes in providing gynecological care to women with chronic vaginal infections, vaginal malodor is a common complaint. Not surprisingly, most of these women have either undiagnosed BV or recurrent BV. However, it has been our observation that there is a smaller cohort of women who present to our clinic with complaints of genital malodor, but who have no clear evidence of actual malodor, based on our evaluation, nor of an underlying medical cause for their compaint. These women pose a diagnostic challenge, and the lack of an available explanation or helpful therapies is a source of great frustration to affected patients. Although this may not be true of all patients whom we have seen with VM, we suspect that many could be considered candidates for a diagnosis of ORS. By identifying patients with unexplained VM and comparing them via a case-control study to women who present with documented BV, this retrospective cohort study is a first step to characterize this patient population and help other investigators develop future study protocols for this troubling problem.

\section{Materials and Methods}

The study population was derived from patients seen at the Drexel Vaginitis Center by one of three providers, from April 1, 2008 until December 31, 2015. The Drexel Vaginitis Center is a tertiary care program established to evaluate women with chronic vulvovaginal symptoms. Each patient undergoes a standardized history and evaluation, which includes testing for
BV with Amsel's criteria, yeast with a yeast culture, and when appropriate trichomonas PCR testing. Approximately 700 new patients are evaluated annually.

Subjects were identified via a patient list generated by running a query for new patient visits with a diagnosis code of vaginal odor (ICD-9 code 625.8; ICD-10 N94.89) and without any other diagnostic code, infectious or non-infectious in etiology, including vaginal atrophy. Patients who presented status post treatment for any type of vaginal infection within the 2 weeks prior to their visit at the Vaginitis Center were excluded from the study, as were any patients under the age of 18 , and any patient who had an objective odor, perceived by the clinician, at time of visit. No VM patient had an identifiable odor detected by the clinician, and lack of odor was confirmed by the medical assistant at the time of the visit. A control group was created by identifying the next patient seen in the clinic following a patient identified for our study group who had the diagnosis of bacterial vaginosis (ICD-9 code 616.1; ICD-10 codes: N76.0 Acute vaginitis; N76.1 Subacute and chronic vaginitis; N76.2 Acute vulvitis; and N76.3 Subacute and chronic vulvitis).

A retrospective chart review of the identified subjects and controls was performed. The following clinical variables were evaluated: general demographics (such as age, ethnicity), medical and psychological co-morbidities, history of treatment for vaginal infections, treatments prescribed, number of return visits with the same complaint, and patient reported improvement. The research study was approved by the Drexel University Institutional Review Board.

Continuous variables were expressed as medians and interquartile ranges. Categorical variables were expressed as numbers and percentages. Differences in demographic characteristics, medical/psychological comorbidities, and vulvar symptoms were assessed using chi-square test and Fischer's Exact test for categorical variables. Student's t-test and MannWhitney test were utilized to compare continuous variables. A $p$ value of $<0.05$ was used to denote statistical significance. All analyses were performed using SPSS version 23.0 for Windows.

\section{Results}

Table 1: Patient characteristics by study.

\begin{tabular}{|c|c|c|}
\hline Variable & VM (N=50) & BV (N=51) \\
\hline Age, median (IQR) & $36.5(27.0,44.0)$ & $128.0,39.0)$ \\
\hline White & Race, $\mathbf{n}(\%)$ & $315(29.4)$ \\
\hline African American & $20(40.0)$ & $5(60.8)$ \\
\hline Other & $27(54.0)$ & $16(31.4)$ \\
\hline Married, $n$ (\%) & $3(6.0)$ & $27.6(23.4,31.1)$ \\
\hline Body mass index (BMI), median (IQR) & $11(22.0)$ & $4(7.8)$ \\
\hline Diabetes & $28.9(22.5,36.4)$ & $4(7.8)$ \\
\hline Hypertension & Medical Comorbidities, n (\%) \\
\hline Irritable bowel syndrome (IBS) & $1(2.0)$ & $1(2.0)$ \\
\hline
\end{tabular}


Journal of Gynecology and Women's Health

\begin{tabular}{|c|c|c|}
\hline Gastroesophageal reflux disease (GERD) & $4(8.0)$ & $1(2.0)$ \\
\hline \multicolumn{3}{|c|}{ Psych Comorbidities, n (\%) } \\
\hline Depression & $1(2.0)$ & $3(5.9)$ \\
\hline Generalized anxiety & $3(6.0)$ & $0(0.0)$ \\
\hline Bipolar & $0(0.0)$ & $2(3.9)$ \\
\hline One or more psych comorbidity & $4(8.0)$ & $4(7.8)$ \\
\hline \multicolumn{3}{|c|}{ Sexual History } \\
\hline Sexually active, n (\%) & $33(66.0)$ & $38(76.0)$ \\
\hline Coitarche (years), median (IQR) & $16(15.0,18.0)$ & $17(15.0,18.5)$ \\
\hline $\begin{array}{l}\text { Total number of lifetime partners, median } \\
\text { (IQR) }\end{array}$ & $5(3.0,10.0)$ & $5.5(3.3,10.8)$ \\
\hline Current number of partners, median (IQR) & $1(0.0,1.0)$ & $1(1.0,1.0)$ \\
\hline \multicolumn{3}{|c|}{ Sex of Partners, $n$ (\%) } \\
\hline Male & $49(98.0)$ & $50(98.0)$ \\
\hline Female & $1(2.0)$ & $0(0.0)$ \\
\hline Both & $0(0.0)$ & $1(2.0)$ \\
\hline \multicolumn{3}{|c|}{ Social History, n (\%) } \\
\hline Tobacco use & $10(20.0)$ & 7 (13.7) \\
\hline Alcohol use & $29(58.0)$ & $25(49.0)$ \\
\hline History of abuse, n (\%) & $2(4.0)$ & $2(3.9)$ \\
\hline \multicolumn{3}{|c|}{ Gynecologic History, n (\%) } \\
\hline Menopause & $8(16.0)$ & $5(9.8)$ \\
\hline Hysterectomy & $6(12.0)$ & $0(0.0)$ \\
\hline \multicolumn{3}{|c|}{ STD History, n (\%) } \\
\hline Gonorrhea/chlamydia & $7(14.0)$ & $11(21.6)$ \\
\hline Trichomonas & $5(10.0)$ & $5(9.8)$ \\
\hline Herpes & $1(2.0)$ & $3(5.9)$ \\
\hline \multicolumn{3}{|c|}{ OB History } \\
\hline Gravida, median (IQR) & $2(0.0,4.0)$ & $1.0(0.0,3.0)$ \\
\hline Parity, median (IQR) & $1(0.0,2.0)$ & $1.0(0.0,2.0)$ \\
\hline
\end{tabular}

VM: Vaginal malodor

BV: Bacterial vaginosis

IQR: Interquartile range

Table 2: Patient symptoms by study group.

\begin{tabular}{|c|c|c|}
\hline Variable & VM (N=50) & BV $(\mathrm{N}=51)$ \\
\hline Age of onset, n (\%) & $27(20.0,40.5)$ & $30(24.0,37.0)$ \\
\hline Symptom duration (years), median (IQR) & $2(1.0,5.5)$ & $1(1.0,2.0)$ \\
\hline \multicolumn{3}{|l|}{ Vulovaginal symptoms } \\
\hline Odor, $\mathrm{n}(\%)$ & $50(100.0)$ & $38(74.5)$ \\
\hline Sweat*, n (\%) & $10(20.0)$ & $0(0.0)$ \\
\hline Fish odor, n (\%) & $23(46.0)$ & $20(39.2)$ \\
\hline Ammonia, n (\%) & $3(6.0)$ & $1(2.0)$ \\
\hline Fried onions, n (\%) & $2(4.0)$ & $0(0.0)$ \\
\hline Garbage, n (\%) & $2(4.0)$ & $0(0.0)$ \\
\hline Others notice odort, n (\%) & $10(20.0)$ & $0(0.0)$ \\
\hline Discharge, $\mathrm{n}(\%)$ & $38(76.0)$ & $45(88.2)$ \\
\hline Irritation, n (\%) & $14(28.0)$ & $28(54.9)$ \\
\hline Itching, n (\%) & $5(36.0)$ & $23(51.1)$ \\
\hline
\end{tabular}


Journal of Gynecology and Women's Health

\begin{tabular}{|c|c|c|}
\hline Dyspareunia, $\mathrm{n}(\%)$ & $5(10.6)$ & $12(24.5)$ \\
\hline Discharge (0-3), median (IQR) & $1.5(0.8,2.0)$ & $0.0(0.0,2.0)$ \\
\hline Irritation (0-3), median (IQR) & $0.0(0.0,1.0)$ & $0.0(0.0,2.0)$ \\
\hline Itching (0-3), median (IQR) & $0.0(0.0,1.0)$ & $0.0(0.0,0.5)$ \\
\hline Dyspareunia (0-3), median (IQR) & $0.0(0.0,0.0)$ & $40(78.4)$ \\
\hline Previously diagnosed BV‡, $\mathrm{n}(\%)$ & $46(92.0)$ & $3(0.0,6.0)$ \\
\hline $\begin{array}{c}\text { Number of times treated in past year, median } \\
\text { (IQR) }\end{array}$ & $3(1.0,6.0)$ & $35(68.6)$ \\
\hline Previously diagnosed yeast, $\mathrm{n}(\%)$ & $28(57.1)$ & $1(0.0,4.0)$ \\
\hline $\begin{array}{c}\text { Number of times treated in past year, median } \\
\text { (IQR) }\end{array}$ & $1(0.0,2.0)$ & \\
\hline
\end{tabular}

2x2 Fisher's Exact Test (Sweat Smell versus No Sweat Smell) x (BV versus VM); $\mathrm{p}=0.001$

$\dagger 2 \times 2$ Fisher's Exact Test (Others Notice Odor versus No Others notice Odor) x (BV versus VM); $p=0.001$

$\ddagger 2 \mathrm{x} 2$ chi-square (Previously diagnosed BV versus No previously diagnosed BV) $\mathrm{x}$ (BV versus VM); $\mathrm{p}=0.055$

A total of 50VM cases and 51BV controls were identified. Table 1 shows the demographic factors, medical/obstetrical history and health characteristics of the cases and controls. There were no significant differences between cases and controls in terms of age, race, body mass index, medical or psychological co-morbidities, and menopause status. Table 2 shows the patient symptoms divided between the cases and control groups. All of the cases, as expected by the case definition, but only $38(74.5 \%)$ of the BV patients, complained of malodor. Both the cases and controls described their odor as fishy in similar percentages. However, cases were more likely to use a descriptor other than fishy when describing their odor. The most common odors patients described outside of fishy were sweat, ammonia, fried onions, and garbage. Of these alternative odors, VM cases were significantly more likely to compare their odor to a sweat-like odor than the BV controls: $10(20 \%)$ of the

VM cases and 0 of the BV cases either compared their genital odor to a sweaty odor, described their odor as emanating from their genitals and armpits, or described a worsening of the odor in the setting of vigorous activity as indicated as the descriptor "sweat" $(\mathrm{P}=0.001)$. Additionally, $10(20 \%)$ of the cases and 0 of the controls complained that others noted the disturbing odor $(\mathrm{P}=0.001)$. Cases and controls complained of additional symptoms associated with vaginal infections (discharge, vulvar itching, irritation) in similar numbers and rated these associated symptoms similarly in terms of severity. The median duration of symptoms was similar in both groups. Both cases and controls reported a high percentage of previous diagnosis and treatment for $\mathrm{BV}, 46(92 \%)$ vs. $40(78.4 \%)$, though cases reported a prior diagnosis of $\mathrm{BV}$ at a greater percentage than BV controls with the $P$ value approaching significance when cases were compared to controls $(\mathrm{p}=0.55)$.

Table 3: Diagnostic characteristics.

\begin{tabular}{|c|c|}
\hline Variable & VM (N=50) \\
\hline Diagnostic testing & $\mathrm{n}(\%)$ \\
\hline Positive whiff test & $0(0.0)$ \\
\hline $\mathrm{pH} \geq 4.5$ & $9(18.4)$ \\
\hline PMN epi cell ratio $>1: 1$ & $2(4.2)$ \\
\hline Positive for Clue cells & $0(0.0)$ \\
\hline Positive for Parabasal cells & $0(0.0)$ \\
\hline Fungal elements (spores/hyphae) & $0(0.0)$ \\
\hline Positive yeast culture & $1(2.0)$ \\
\hline
\end{tabular}

Table 3 shows the diagnostic testing performed on the malodor patients. No VM patient had an identifiable odor detected by the clinician, nor did any have a positive amine ("whiff") test. For the BV control group, all had 3 out of 4 Amsel's criteria to be diagnosed with BV (data not shown). Among the cases, none met 3 out of 4 Amsel's criteria for BV, although 9 $(18.4 \%)$ had a vaginal $\mathrm{pH}>4.5$. In terms of yeast diagnoses, no cases had fungal elements noted on wet mount, but $1(2 \%)$ had a positive yeast culture. Additionally, 2 (4.2\%) cases had a PMN epi cell ratio $>1: 1$ on saline microscopy; none had parabasal cells. None of the women with a $\mathrm{pH}>4.5$ exhibited signs or symptoms of vaginal atrophy.

Thirty-one (63.3\%) women attempted treatment of some kind, either the Waterworks $\AA$ douche apparatus (Abbott Research Group, Pittsburgh, Pennsylvania) 11(22.4\%), switching to an antibacterial deodorant soap $26(53.1 \%)$, or applying a deodorant to the genital area $6(12.2 \%) .28(56 \%)$ of the women returned for a follow up visit with a median of 2 visits. $18(78.3 \%)$ of the women reported no improvement (defined as less than $25 \%$ subjective improvement), 3 (13\%) reported some improvement and $2(8.7 \%)$ of the women reported cure (defined as a $80 \%$ or greater subjective improvement score). Of the 16 with follow-up visits, 2/6 reported some improvement and 1 described herself as cured with the Waterworks ${ }^{\circledR}$ device. 
Improvement with antibacterial/deodorant soaps and genital deodorants were minimal.

\section{Comments}

$\mathrm{BV}$ is the most common cause of vaginal malodor and the most common cause of infectious vaginitis. However it is estimated that one third of women presenting with a primary complaint of vaginal odor have no objective cause [1]. Although this complaint may seem like a mundane topic which is not worthy of serious investigation, complaints of vaginal malodor can result in significant dissatisfaction and frustration for patient and clinician alike. To date there is no information on the incidence of vaginal malodor without an infectious etiology nor is there demographic information on this commonly seen patient. Furthermore, VM is a difficult condition to diagnose as it is a diagnosis of exclusion, often requiring multiple return visits. Due to the ambiguity of this complaint, many providers may tend to just assume that these women have BV based on chief complaint alone. One study noted that over half the women seen in a specialty vaginitis center were started on treatment before a complete office based work-up was preformed and that a Whiff test and pH test were only preformed in 3\% of office visits [9]. Indeed, most of the VM cases in this study were treated for BV prior to seeking care at the Drexel Vaginitis Center. To our knowledge, this study is the largest study identifying women with vaginal malodor presenting to a tertiary care vaginitis center, where extra effort and expertise is placed to diagnose underlying infections or causes for abnormal odor. We identified possible demographic differences between women who present with VM and those who present with BV and found them, surprisingly, to be fairly similar to one another.

There were two features that were statistically significant between VM and BV patients: the belief that others smelled the odor and the description of the odor. VM patients were more likely to describe their odor as something other than fishy in nature and were more likely to associate their odor with sweat, complaining of either a sweaty odor, describing their odor as emanating from their genitals and armpits, or describing a worsening of the odor in the setting of vigorous activity. Our results suggest that if a patient presents with the complaint of vaginal odor that smells of or worsens with sweat, describes the odor as a foul odor other than fishy in nature, or believes that other people can smell their offending odor, they are less likely to have an infectious etiology causing their VM. Our results show that few patients with VM are helped with interventions that might help patients with chronic malodor, including the Waterworks $®$ device which is an FDA approved treatment for vaginal malodor which in initial studies may improve odor in the setting of noninfectious VM [10].

At the time that patients with VM were being seen in the office, we did not know that we would be doing a retrospective analysis of this patient population. Thus, there are elements of the patient histories which were not captured in our chart review but which speak to the burden carried by women with chronic VM. Of the patients with chronic VM, all were motivated enough by this problem that they had sought treatment from many providers prior to being referred to our practice. Anecdotally, they spoke eloquently of how this perception of VM had ruined their quality of life and fueled an intense frustration. Some women had quit their jobs or refused to go to the lunchroom or bathroom when at work because they were afraid that their coworkers would comment on the odor. Others avoided entering into new relationships because of embarrassment. We saw grandmothers who would no longer see their grandchildren because they were sure that the child would notice a smell if they sat next to them or were even in the same room. One patient was so upset that we would not prescribe metronidazole for her that she contacted multiple individuals high up in our institution's administrative hierarchy to request that we be instructed to prescribe it for her, even though she had had an estimated 7-8 BV treatment courses in the year prior to seeing us with no effect on the odor. Thus, what our study data fail to capture are measures of quality of life which would demonstrate the impact that chronic VM has on affected women.

Olfactory Reference Syndrome (ORS) is a little known psychiatric disorder characterized by a preoccupation with the belief that the affected individual emits a foul and offensive body odor, often from the genital region $[5,6,7,8]$. While this condition is almost completely unknown within the gynecologic world, what little data exist on the condition suggest that affected individuals often present to non-psychiatric clinicians for help $[6,7]$. It is possible that many women presenting to their OB/GYN with the complaint of a non-existent genital odor, potentially meet criteria for ORS, if the clinician knew its signs and symptoms. Experts suggest three criteria to help screen for ORS: 1) patient presents with an odor that is not perceived by others, often with the belief that others can perceive and are troubled by the odor, 2) the odor causes significant distress and impairment, and 3) symptoms cannot be attributed to any other mental or medical cause [5]. Additionally, researchers in ORS noted that this population tends to complain of both a bodily odor (bad breath or sweat) in addition to what they described as a "nonbodily odor" such as ammonia, fish, and rotten onions [5,6,7].

Similar to the ORS population described in the psychiatric literature, our VM population significantly described their odor as a "sweat" like odor, either describing their odor as sweaty, complaining of the same odor emanating from their genitals and their armpits, or describing a worsening of the odor in the setting of vigorous activity. They were also more likely to use descriptors other than fish to describe their odor, and these odor descriptors were consistent with the psychiatric literature's descriptions of perceived odors in ORS patients. Furthermore, our VM patients were more likely to complain that others smelled their odor, although no objective odor was perceived. Interventions to help the odor were generally unhelpful. If these patients do in fact suffer from ORS, it is possible that it could have been triggered by having BV at one point in their life. Regardless, if suspicious for ORS, psychiatric experts note that attempting to convince 
these patients that they do not have an odor is futile and anxiety provoking. Rather, they recommend referral to psychiatric clinicians who can initiate a combination of therapy with SSRIs and cognitive behavior therapy to help patients focus on the distress of the perceived odor, rather than the odor itself [6].

Women with VM remain poorly understood and are often treated for BV due to presenting symptoms alone without any symptomatic relief. With this study, we would like to emphasize that not all women with VM have BV, and that many women with VM are overtreated for BV. However, since the patients with VM had received many treatments prior to our evaluation, we acknowledge that some of the VM patients may have had BV prior to seeing us. Indeed $8(16.3 \%)$ of patients were diagnosed with BV at one of their return visits, although many continued to come back after treatment for BV with a complaint of continued VM with no objective BV findings, thus suggesting that BV could have been an incidental finding to the chronic complaint of VM. Furthermore, since odor is a subjective complaint, it is possible that some of the VM patients did have an abnormal odor which the clinicians were unable to detect; if so, the lack of response to various interventions speaks to how difficult it is to improve chronic VM. We feel that future studies of women with noninfectious VM should focus on characterizing the odor in a more detailed manner, obtaining more information, optimally with validated questionnaires, on the effects of this condition on quality of life, self-image, and relationships. Since some of the affected women may have ORS, referral for psychological evaluation and possibly cognitive behavioral therapy should be considered in patients with VM.

\section{Disclosures}

AM, CM, and KTS have nothing to disclose. PN has no conflict with regard to this study, but reports consulting arrangements with Viamet, Symbiomix Therapeutics, Cidara Therapeutics, and Exeltis, USA; his institution has received research grants from Viamet Pharmaceuticals, Novadigm Therapeutics, Alfa Wassermann S.p.A., Cidara Therapeutics, Curatek Pharmaceuticals and Symbiomix Therapeutics. This paper was presented as an oral presentation at the 24th World Congress of the International Society for the Study of Vulvovaginal Diseases in Mendoza, Argentina, September 11-15, 2017.

\section{Condensation}

Women with vaginal malodor are demographically similar to women with recurrent bacterial vaginosis. Standard interventions to address odor are frequently unhelpful, suggesting a psychiatric etiology. The purpose of this study is to make clinicians aware that there exists a group of women with noninfectious chronic vaginal malodor (VM) who have not been helped by the medical community. Although a seemingly mundane problem, this study is, to our knowledge, the first to take a serious look at this population and compare them to women with recurrent bacterial vaginosis infections (BV). While demographically similar, VM subjects differed from BV counterparts in their description of the odor and in their perception that others smelled the odor, which anecdotally, greatly impacted quality of life. Some of these women may have a psychiatric disorder, olfactory reference syndrome. We feel our study will help gynecologists think of a condition which they probably encounter but of which they have never heard.

\section{References}

1. Subramanian C, Nyirjesy P, Sobel JD (2012) Genital malodor in women: a modern reappraisal. J Low Genit Tract Dis 16(1): 49-55.

2. Landers DV, Wiesenfeld HC, Phillip HR, Krohn MA, Hillier SL, et al. (2004) Predictive value of the clinical diagnosis of lower genital tract infection in women. Am J Obstet Gynecol 190(4): 1004-1010.

3. Anderson MR, Klink K, Cohrssen A (2004) Evaluation of vaginal complaints. JAMA 291(11): 1368-1379.

4. Schaaf V, Perez-Stable EJ, Borchardt K (1990) The limited value of symptoms and signs in the diagnosis of vaginal infections. Arch Intern Med 150(9): 1929-1933.

5. Feusner JD, Phillips KA, Stein DJ (2010) Olfactory Reference Syndrome: Issues for DSM-V. Depress Anxiety 27(6): 592-599.

6. Phillips KA, Menard W (2011) Olfactory reference syndrome: demographic and clinical features of imagined body odor. Gen Hosp Psychiatry 33(4): 398-406.

7. Greenberg JL, Shaw AM, Reuman L, Schwartz R, Wilhelm S, et al. (2016) Clinical features of olfactory reference syndrome: An internet-based study. J Psychosom Res 80: 11-16.

8. Stein DJ, Roux LL, Bouwer C, Heerden VB (1998) Is Olfactory Reference Syndrome an Obsessive-Compulsive Spectrum Disorder? The Journal of Neuropsychiatry and Clinical Neurosciences 10(1): 96-99.

9. Wiesenfeld HC, Macio I (1999) The infrequent use of office-based diagnostic tests for vaginitis. Am J Obstet Gynecol 181(1): 39-41.

10. Hassan S, Chatwani A, Brovender H, Zane R, Valaoras T, et al. (2011) Douching for Perceived Vaginal Odor with No Infectious Cause of Vaginitis: A Randomized Controlled Trial. J Low Genit Tract Dis 15(2): 128-133.

11. Doty R, Ford M, Preti G, Huggins G (1975) Changes in the intensity and pleasantness of human vaginal odors during the menstrual cycle. Science 190(4221): 1316-1318. 
(c) This work is licensed under Creative

\section{Your next submission with Juniper Publishers} will reach you the below assets

- Quality Editorial service

- Swift Peer Review

- Reprints availability

- E-prints Service

- Manuscript Podcast for convenient understanding

- Global attainment for your research

- Manuscript accessibility in different formats

( Pdf, E-pub, Full Text, Audio)

- Unceasing customer service

Track the below URL for one-step submission https://juniperpublishers.com/online-submission.php 